\title{
Preparation and evaluation of spirulina polysaccharide nanoemulsions
}

\author{
BINGYUE WANG $^{1 *}$, TIANGE CAI ${ }^{2 *}$, QIAN LIU $^{3 *}$, JOHN CAMERON COLE WHITNEY $^{4}$, MANLING DU $^{1}$, \\ QIANQIAN MA ${ }^{1}$, RONGHUA ZHANG ${ }^{1}, \mathrm{LI} \mathrm{YANG}^{1}$, $\mathrm{SUSAN} \mathrm{P.C.COLE}^{5}$ and YU CAI ${ }^{1,6}$ \\ ${ }^{1}$ College of Pharmacy, Jinan University, Guangzhou, Guangdong 510632; ${ }^{2}$ College of Life Sciences, \\ Liaoning University, Shenyang, Liaoning 110000; ${ }^{3}$ Guangzhou Jiayuan Pharmaceutical Technology Co., \\ Ltd., Guangzhou, Guangdong 510663, P.R. China; ${ }^{4}$ Department of Biochemistry and Biomedical Sciences, \\ McMaster University, Hamilton, ON L8S 4K1; ${ }^{5}$ Division of Cancer Biology and Genetics, Queen's University, \\ Kingston, ON K7L 3N6, Canada; ${ }^{6}$ Cancer Institute of Jinan University, Guangzhou, Guangdong 510632, P.R. China
}

Received January 23, 2018; Accepted May 17, 2018

DOI: $10.3892 /$ ijmm.2018.3717

\begin{abstract}
The aim of the present study was to prepare spirulina polysaccharide (PSP) into an oral nanoemulsion (NE) with the aim of improving its oral bioavailability and prolonging its sustained release effect. The PSP-NE was prepared through a phase transformation method, and its formulation components were screened through the use of a pseudo-ternary phase diagram. The optimal formulation of PSP-NE was determined to be: $11.9 \%$ Span $80,6.0 \%$ Tween$80,9.0 \%$ ethanol, $62.8 \%$ soybean oil, and $10.3 \%$ PSP aqueous solution. The prepared PSP-NE was clear and transparent, had a uniform color and spherical morphology, exhibited stability and no adhesion. The average particle size was $79.93 \pm 19 \mathrm{~nm}$, the polydispersity index was $0.185 \pm 0.04(n=3)$, and the entrapment rate was $62 \%$. Small-animal imaging results showed that the prepared PSP-NE exhibited a sustained release and tissue effect in contrast to the PSP aqueous solution. The present study showed that the prepared PSP-NE not only exhibited a sustained release and tissue effect in contrast to the PSP aqueous solution, but also had superior performance in terms of antitumor and antioxidant effects.
\end{abstract}

Correspondence to: Dr Yu Cai, College of Pharmacy, Jinan University, 601 Huangpu Avenue West, Guangzhou, Guangdong 510632, P.R. China

E-mail: caiyu8@sohu.com

Dr Susan P.C. Cole, Division of Cancer Biology and Genetics, Queen's University, 10 Stuart Street, Kingston, ON K7L 3N6, Canada

E-mail: sp.cole@queensu.ca

"Contributed equally

Key words: spirulina polysaccharide, nanoemulsion, sustained release, tissue specificity, antitumor, antioxidant, pseudo-ternary phase diagram

\section{Introduction}

Spirulina polysaccharide (PSP), a type of water-soluble, physiologically active polysaccharide extracted from spirulina, has a large and complex molecular structure, which is mainly composed of glycosidic bonds (1). PSP is reported to have an effect on inhibiting tumor cell growth through inhibiting the synthesis of nucleic acid and proteins in cancer cells, but not directly killing cancer cells. In addition, the inhibitory effect of PSP on cancer cells has been reported to be time-dependent $(2,3)$. It is well known that free radicals can oxidize biomolecules and are important in several degenerative and pathological processes $(4,5)$. As an antioxidant, PSP can maintain cellular health and inhibit senescence in the body by removing excess free radicals and preventing the oxidation of cellular oxidative substrates $(6,7)$. PSP can enhance the non-specific cellular immune function in the body, and improve the ability to resist the invasion of viruses (8). Therefore, it has potential application and development value.

Nanoemulsions (NEs) (9) are formed spontaneously by mixing together an aqueous phase, an oil phase, surfactant, and co-surfactant; can be a thermodynamically stable, isotropic, transparent, or translucent homogeneous dispersion; they have a particle size of 1-100 nm. NEs are stabilized and not layered by autoclaving and high-speed centrifugation (10). NEs have been used since 1930, particularly in floor polishing products, including liquid waxes, fuels and dry lotions (11). At present, $\mathrm{NE}$ technology is employed in the production of commonly used chemicals, specialized chemicals, and in the petroleum and materials science $(12,13)$, biotechnology and pharmaceutical industries (14). Furthermore, NEs are novel drug carriers and offer numerous beneficial properties (15). In particular, they exhibit thermodynamic stability and low viscosity, and are thus easy to prepare and store. They also increase the solubility of fat-soluble drugs.

The pseudo-ternary phase diagram method is always applied to reflect the mutual change of a three-component system on a plane triangle under isothermal isobaric conditions. In NEs, the three vertices in the pseudo-ternary phase diagram respectively represent the aqueous phase, the oil 
phase, and the surfactant. The ratio between two components is determined by the distance from this point to the endpoints on both sides, and the proportional relationship between the three components is within the pseudo-ternary phase diagram. This method turns the association among the three components in the NE formation into a flat graph, which is intuitive, concise and enables easy determination of the proportion of each component.

Studies have indicated that a PSP-loaded NE offers a wide application in clinical use, including increasing stability and sustained drug release. Furthermore, a PSP-loaded NE has been shown to improve clinical medication safety by reducing its irritant or toxic side effects, enhancing its availability and bioavailability by avoiding destruction when passing through the digestive tract, and increasing specificity to drug targeting by extending the release time of water-soluble drugs. In addition to achieving the above effects, a PSP-loaded NE can mask strong odors to improve patient compliance $(16,17)$. Therefore, the fish-flavored PSP was encapsulated into NE to prepare PSP-NE, which aimed to improve the oral bioavailability and stability of PSP, as well as the antitumor and antioxidative effects.

\section{Materials and methods}

Materials. The PSP (polysaccharide content of 71.65\%) produced by the Department of Traditional Chinese Medicine Department of Jinan University (Guangzhou, China) was a spirulina lye extract product, and the monosaccharide composition was as follows: Glucose $(21.3 \pm 1.4 \%)$, rhamnose $(43.6 \pm 2.7 \%)$, xylose $(2.4 \pm 0.6 \%)$, galactose $(1.3 \pm 0.2 \%)$ and arabinose $(1.1 \pm 0.1 \%)$. Other reagents included indocyanine green (MedChem Express, Monmouth Junction, NJ, USA), 1,9-dimethylmethylene blue (DMB Sigma; EMD Millipore, Billerica, MA, USA), Span 80 (Aladdin Reagent Co., Ltd., Shanghai, China), Tween-80 (Aladdin Reagent Co., Ltd.), injection-grade soybean oil (Emerging Tieling Pharmaceutical Co., Ltd., Tieling, China), 3-(4,5-dimethyl-2-thiazolyl)-2,5-diphenyl-2-H-tetrazolium bromide (MTT; Sigma; EMD Millipore), RPMI-1640 medium (Gibco; Thermo Fisher Scientific, Inc., Waltham, MA, USA), fetal bovine serum (FBS; Gibco; Thermo Fisher Scientific. Inc.) Sudan Red III, methylene blue, dimethyl sulfoxide, ethanol, iron sulfate, isopropanol, n-butanol, n-octanol and polyethylene glycol 400 (Tianjin Daming Chemical Reagent Factory, Tianjin, China).

Experimental animals and tumor cell line. A total of 9 BALB/c-nu mice (age: 8 weeks; weight: $22 \mathrm{~g}$ ) with equal numbers of males and females were provided by Beijing Huafu Kang Biotechnology Co., Ltd. (Beijing, China), and were maintained in our facility with free access to water and food, under a 12-h light/dark cycle, with $40 \%$ humidity. The HepG2 human hepatoblastoma cell line and MCF-7 human breast adenocarcinoma cell line were obtained from Jiangsu KeyGen Biotech Co., Ltd., (Jiangsu, China).

Surfactant preparation. The NEs were prepared with a high-speed homogenizer. For formulation screening, a surfactant or mixed surfactant was selected according to the NE type declared. A low hydrophile-lipophile balance (HLB) value $(<8)$ was used for the preparation of a water-oil $(\mathrm{W} / \mathrm{O}) \mathrm{NE}$ and a high HLB value $(>10)$ was used for an oil-water $(\mathrm{O} / \mathrm{W})$ NE. As PSP was readily soluble, the PSP was mixed with the water-in-oil NE.

\section{Screening of PSP-NE components}

Combination of surfactants. The effects of seven complexes of mixed surfactants (Span 80 and glycerolmonooleate at ratios of 3:2 and 3:4; glycerolmonooleate and Tween-80 at ratios of 2:1, 3:1 and 4:1, and Span 80/Tween-80 at ratios of 3:1 and 4:1) were investigated on the NE-forming area of a pseudo-ternary phase diagram in the surfactant/anhydrous ethanol/soybean oil/water system [surfactant and co-surfactant ratio $\left(K_{\mathrm{m}}\right)$ of 2:1] at room temperature (18).

Screening of co-surfactants. At room temperature, the effects of five co-surfactants (ethanol, isopropanol, polyethylene glycol 400, n-butanol and n-octanol) on the NE-forming area of the pseudo-ternary phase diagram were investigated in a Span 80/Tween-80/co-surfactant/soybean oil/water system $\left(K_{\mathrm{m}} 2: 1\right)$.

Effect of $K_{m}$ on the formation of NEs. The effects of $K_{\mathrm{m}}$ values at 2:1,3:1 and 4:1 on the NE-forming area of the pseudo-ternary phase diagram were investigated in a Span 80/Tween-80/anhydrous ethanol/soybean oil/water system at room temperature.

Screening of oil phase. Liquid paraffin, medium-chain fatty acid triglyceride, soybean oil and sesame oil were used to determine whether they have individual effects on the NE-forming regions. This step was performed on a Span 80/Tween-80/anhydrous ethanol/oil phase/water $\left(K_{\mathrm{m}} 2: 1\right)$ system at room temperature.

Screening of the aqueous phase. The individual effects of 1 , 2, 5, 10 and 20\% PSP aqueous solutions on the NE-forming areas were investigated in a Span 80/Tween-80/anhydrous ethanol/soybean oil/PSP aqueous solution system $\left(K_{\mathrm{m}} 2: 1\right)$ at room temperature.

Titration to construct the pseudo-ternary phase diagram. The pseudo-ternary phase diagram was drawn by titration. For the determination of the optimal prescription as the main evaluation index for the NE formation, the mixed (Smix), oil and aqueous phases were used as the three vertices of the pseudo-ternary phase diagram on the basis of the size of the NE-forming area $(19,20)$.

\section{Evaluation of NE quality}

Appearance and type. The appearance of the prepared NE with respect to clarity, transparency and homogeneity was examined macroscopically. The type of NE was determined by using different modes of the spreading speed of methylene blue (water-soluble dye) and Sudan red (oil-soluble dye) (21).

Shape and particle size. The shape was observed using transmission electron microscopy (TEM; Tecnai 10; Philips Healthcare, DA Best, The Netherlands) following diluting of the NE with soybean oil. The particle size and polydispersity 
index (PDI) of the prepared NE were measured with a laser particle size analyzer (Nano ZS 90; Malvern Instruments, Inc., Westborough, MA, USA) (22).

Viscosity and $\mathrm{pH}$. The viscosities and $\mathrm{pH}$ values of the empty NE and PSP-NE were measured with a viscometer (SV-10 type; A\&D Co., Ltd., Tokyo, Japan) and pH meter (pH 10/100 type, Shanghai Di Yim Instrument Co., Ltd., Shanghai, China), respectively.

Stability. The stability of the NEs were determined through a hot and cold cycle test $\left(4\right.$ and $\left.60^{\circ} \mathrm{C}\right)$, light experiment $(4,500 \pm 500 \mathrm{~lx})$ and accelerated experiment $\left(40 \pm 2^{\circ} \mathrm{C}, 75 \pm 5 \% \mathrm{RH}\right)$.

PSP content in the PSP-NE and entrapment rate. Following extraction from the prepared PSP-NE $(1 \mathrm{ml})$ with a mixed solvent of chloroform and ethanol (1:1), an appropriate volume of acetone was added, and the PSP-NE was then subjected to ultrasonication until breakage. The PSP content in the prepared PSP-NE (C1) was measured by high-performance liquid chromatography (HPLC; Agilent 1260, Agilent Technologies, Inc., Santa, Clara, CA, USA) for refractive index (RI) detection.

The PSP-NE $(1 \mathrm{ml})$ was placed in a $10 \mathrm{ml}$ volumetric flask. An equal volume of acetone was then added. The PSP-NE was subjected to ultrasonication until it breakage and maintained constant at $10 \mathrm{ml}$. The total quantity of PSP (C2) was also measured by HPLC and RI detection. The entrapment rate was calculated according to the following formula: Entrapment rate $(\%)=\mathrm{C} 1 / \mathrm{C} 2 \times 100 \%$.

Distribution of PSP-NE following oral administration. Indocyanine green was dissolved in the PSP aqueous solution (ICG-PSP) and then added to the NE to form an ICG-PSP-NE complex (The final concentration of ICG in both solutions is $0.05 \mathrm{mg} / \mathrm{ml}$ ). A total of $9 \mathrm{BALB} / \mathrm{C}$ nude mice were assigned to each of three experimental groups according to treatment type $(0.1 \mathrm{ml} / 100 \mathrm{~g}$ of ICG-PSP, ICG-PSP-NE, and physiological saline, ig). The mice were subjected to fasting $4 \mathrm{~h}$ following their respective treatment and then placed in a live imaging device (Lumina XR Series III; Perkin-Elmer, Inc., Waltham, MA, USA) to observe fluorescence distribution in vivo at 0.5 , $1,2,4,8,24$ and $36 \mathrm{~h}$.

Antitumor effects of PSP-NE and PSP aqueous solution. The cytotoxicities of PSP and PSP-NE were determined by measuring the inhibition of cell growth using an MTT assay (23). The cells were maintained in RPMI 1640 medium with $10 \%$ FBS and $0.1 \mathrm{mg} / \mathrm{ml}$ penicillin $\mathrm{G}$ and $100 \mathrm{U} / \mathrm{ml}$ streptomycin in a humidified atmosphere of $5 \% \mathrm{CO}_{2}$ at $37^{\circ} \mathrm{C}$. The HepG2 cells and MCF-7 cells were seeded separately on a 96-well plate at a cellular density of 5,000 cells/well when at a confluence of $\sim 80 \%$. Following treatment of the cells with the various concentrations of PSP and PSP-NE over 48 h, $20 \mu \mathrm{l}$ MTT solution was added to the cells. The cell viability was determined at $570 \mathrm{~nm}$ in a microplate reader (Synergy HT; BioTek Instruments, Inc., Winooski, VT, USA). Cell toxicity was calculated according to the following equation: Cell viability $(\%)=\mathrm{Abs}_{\text {sample }} / \mathrm{Abs}_{\text {control }} \mathrm{x} 100 \%$, where $\mathrm{Abs}_{\text {sample }}$ is the absorbance of cells in the presence of different formulations, $\mathrm{Abs}_{\text {control }}$ is the absorbance of cells in the absence of drug.
Table I. pH and viscosity of empty NE and PSP-NE.

\begin{tabular}{lcc}
\hline Sample & $\mathrm{pH}$ & $\begin{array}{c}\text { Viscosity } \\
\left.\text { (relative to water; }{ }^{\circ} \mathrm{E}\right)\end{array}$ \\
\hline NE only & $7.37 \pm 0.03$ & $11.68 \pm 0.03$ \\
PSP-NE & $7.24 \pm 0.02$ & $12.37 \pm 0.06$ \\
\hline
\end{tabular}

PSP, spirulina polysaccharide; NE, nanoemulsion.

Antioxidant effects of PSPNE and PSP aqueous solution 1 1,1-diphenyl-2-picrylhydrazyl radical $(D P P H \bullet)$ scavenging assay. The DPPH• is a stable free radical, which is often used as a tool to estimate the antioxidant capacity. The DPPH free radical scavenging activities of PSP and PSP-NE were measured using DPPH according to a published method with modifications $(7,24)$. Vitamin $\mathrm{C}(\mathrm{Vc})$ was used as a positive control. The sample solution $(2 \mathrm{ml})$, including Vc, PSP or PSP-NE samples at different concentrations (1, 2, 3, 4 and $5 \mathrm{mg} / \mathrm{ml}$ ) was added to a tube containing $2 \mathrm{ml}$ DPPH solution $(0.04 \mathrm{mg} / \mathrm{ml}$ in ethanol). Following incubation in the dark for $20 \mathrm{~min}$ at $37^{\circ} \mathrm{C}$, the absorbance was measured at $517 \mathrm{~nm}$. The DPPH radical scavenging activity (\%) was calculated using the following equation (24): Scavenging activity $(\%)=1-\left(\mathrm{A}_{1}-\mathrm{A}_{2}\right) / \mathrm{A}_{0}$ $\mathrm{x} 100 \%$, where $\mathrm{A}_{0}$ is the absorbance of DPPH solution without samples. $A_{1}$ is the absorbance of DPPH with the samples, and $\mathrm{A}_{2}$ is the absorbance of background solution (distilled water instead of DPPH solution).

Hydroxyl radical $(\mathrm{OH} \bullet)$ scavenging assay. The $\mathrm{OH} \bullet$ scavenging activities of PSP and PSP-NE were measured following the modified method of Wu et al (24). Vc was also used as a reference material for a positive control. The sample solution (1 ml) with different concentrations $(1,2,3,4$ and $5 \mathrm{mg} / \mathrm{ml})$, $1 \mathrm{ml} \mathrm{FeSO}_{4}$ solution $(9 \mathrm{mmol} / \mathrm{l})$ and $1 \mathrm{ml}$ salicylic acid ethanol solution $(9 \mathrm{mmol} / \mathrm{l})$ were fully mixed, and the reaction was started with $1 \mathrm{ml} \mathrm{H}_{2} \mathrm{O}_{2}$ solution $(8 \mathrm{mmol} / \mathrm{l})$. The absorbance of the mixture solution was measured at $510 \mathrm{~nm}$ following incubation at $37^{\circ} \mathrm{C}$ for $40 \mathrm{~min}$. The $\mathrm{OH} \bullet$ scavenging activity was calculated as follows: Scavenging activity $(\%)=1-\left(\mathrm{A}_{1}-\mathrm{A}_{2}\right) / \mathrm{A}_{0}$ $\mathrm{x} 100 \%$, where $\mathrm{A}_{0}$ is the absorbance of the negative control (without samples), $A_{1}$ is the absorbance of the sample mixture, and $A_{2}$ is the absorbance of background solution (distilled water instead of $\mathrm{H}_{2} \mathrm{O}_{2}$ ).

Statistical analysis. Quantitative data are expressed as the mean \pm standard deviation. Statistical analysis (one-way analysis of variance) was performed with GraphPad Prism. V6.0 software (GraphPad Software, Inc., La Jolla, CA, USA). P<0.05 was considered to indicate a statistically significant difference.

\section{Results}

\section{Optimization of the PSP-NE formulation}

Surfactant complexes. The seven ratios of surfactant complexes all had good emulsifying ability to form NEs. The results of the pseudo-ternary phase diagram are shown in Fig. 1. The largest NE-forming area was observed at Span 80/Tween-80=3:1, and 

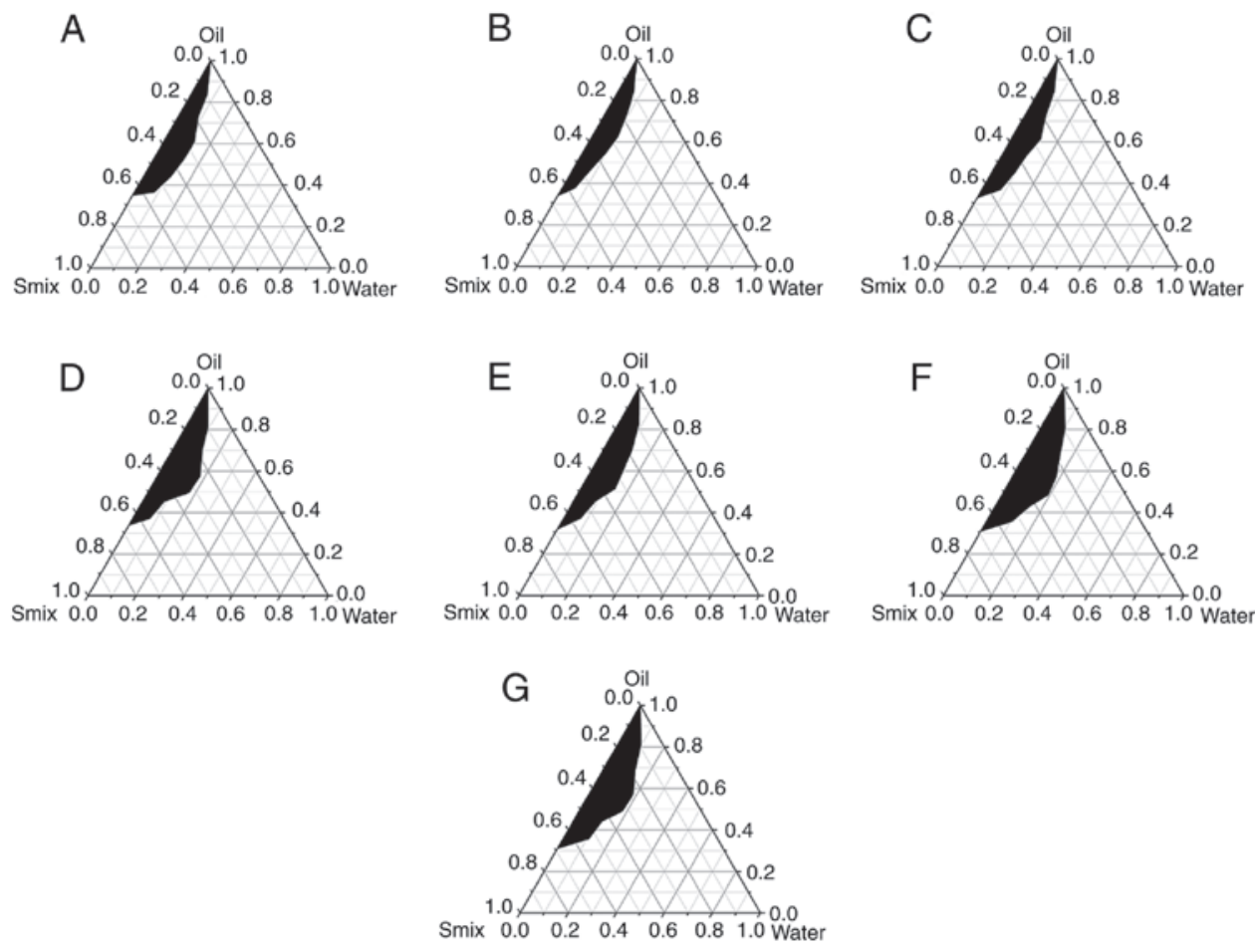

Figure 1. Pseudo-ternary phase diagrams of mixed surfactants. (A) Span 80/glyceryl monooleate ratio of 3:2; (B) glyceryl monooleate/Span 80 ratio of 4:3; (C) glycerylmonooleate/Tween-80 ratio of 4:1; (D) Span 80/Tween-80 ratio of 4:1; (E) glyceryl monooleate/Tween-80 ratio of 2:1; (F) Span 80/Tween-80 ratio of 3:1; (G) glyceryl monooleate/Tween-80 ratio of 3:1. Smix, surfactant mix.

this ratio was selected as the optimal surfactant complex for subsequent experiments.

Screening of co-surfactant and $K_{m}$. Among the several commonly used co-surfactants, only anhydrous ethanol formed NE when the $K_{\mathrm{m}}$ values were 4:1,3:1, and 2:1. In addition, it did not significantly change in appearance regardless of storage in either hot or cold temperatures. Therefore, anhydrous ethanol was selected as a co-surfactant. The pseudo-ternary phase diagram drawn by different $K_{\mathrm{m}}$ values is shown in Fig. 2 . The NE-forming area at $c\left(K_{\mathrm{m}}=2: 1\right)$ was the largest, and was selected for subsequent experiments.

Oil phase screening. Four oil phases were assessed for their ability to form NE and the results are shown in Fig. 3. The NE-forming areas were as follows: Soybean oil $>$ liquid paraffin $>$ medium-chain fatty acid triglycerides $>$ sesame oil. Therefore, soybean oil was selected as the preferred oil phase for subsequent experiments.

Aqueous phase screening. PSP solution at $<20 \%$ had no significant effect on NE formation. At higher concentrations, the NE became turbid. As shown in the pseudo-ternary phase diagram (Fig. 4), the NE-forming area was as follows: $1>2>5$ $>10>20 \%$ PSP. There were no significant differences among the areas at 1,2 and 5\% PSP, however, the NE formation was considerably reduced when PSP concentration exceeded $10 \%$. PSP at $10 \%$ was the final water phase used in consideration of the drug loading.

Therefore, the final NE formulation consisted of $11.9 \%$ Span $80,6.0 \%$ Tween-80, 9.0\% absolute ethanol, $62.8 \%$ soybean oil, and $10.3 \%$ aqueous PSP.
Characteristics of the PSP-NE formulation

Appearance and type identification. The PSP-NE formed according to the above formulation was light yellow, transparent and uniform. Following high-speed centrifugation, the NE remained in a single phase, and the diffusion rate of the Sudan red exceeded that of the methylene blue (Fig. 5). These observations demonstrated that the PSP-NE was a W/O NE.

Morphological observation and particle size determination. The PSP-NE droplets were homogeneous, spherical and non-adhesive. As shown in Fig. 6, the average particle sizes were $79.93 \pm 19 \mathrm{~nm}$, and the PDI values were $0.185 \pm 0.04(n=3)$.

Viscosity and $\mathrm{pH}$. The viscosity and $\mathrm{pH}$ values obtained in the determination of empty NE and PSP-NE are shown in Table I.

Stability. The PSP-NE did not exhibit a muddy consistency following stratification or sedimentation, and remained clear and transparent. As indicated in Table II, the results of the light experiment showed that PSP-NE was stable for up to 10 days. As indicated in Table III, the PSP-NE was stable for up to 2 months during the accelerated experiment. Together, these observations demonstrated that PSP-NE was stable.

PSP content in the PSP-NE and entrapment rate. The content of PSP measured was $5.14 \pm 0.06 \mathrm{mg} / \mathrm{ml}$. The entrapment rate measured was $62 \%$.

Biodistribution following oral administration in nude mice. The fluorescence distribution at different time points are shown in Fig. 7A (PSP) and Fig. 7B (PSP-NE). In the PSP 

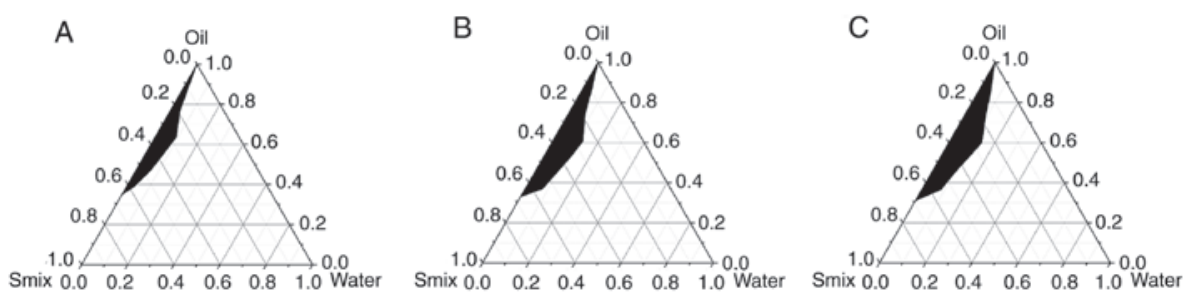

Figure 2. Pseudo-ternary phase diagrams under different $K_{m}$ values. (A) $K_{m}=4: 1$; (B) $K_{m}=3: 1$; (C) $K_{m}=2: 1$. $K_{m}$, surfactant and co-surfactant ratio; Smix, surfactant mix.
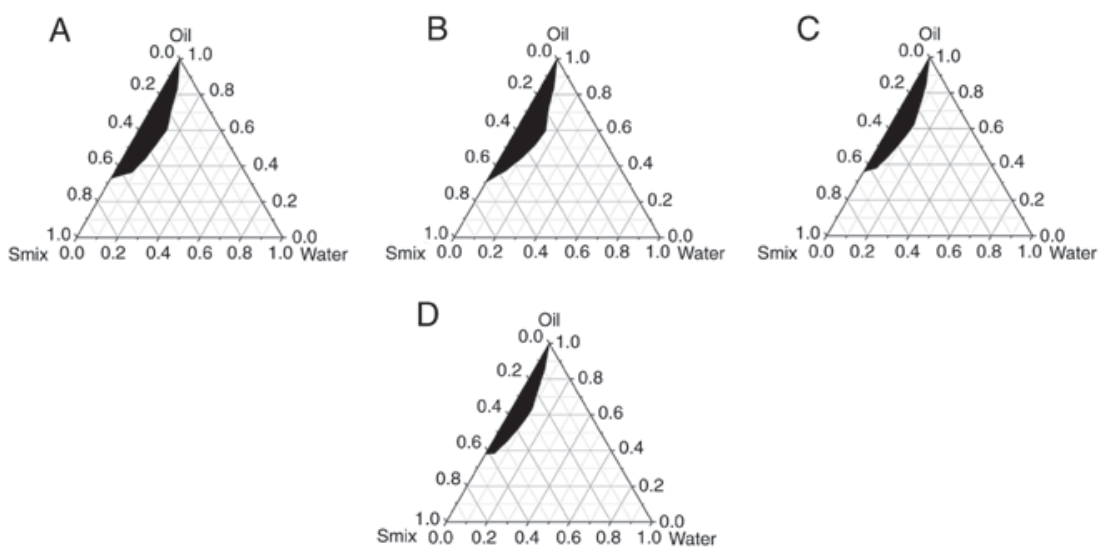

Figure 3. Pseudo-ternary phase diagrams using different oils. (A) Soybean oil; (B) liquid paraffin; (C) medium chain triglyceride; (D) sesame oil. Smix, surfactant mix.
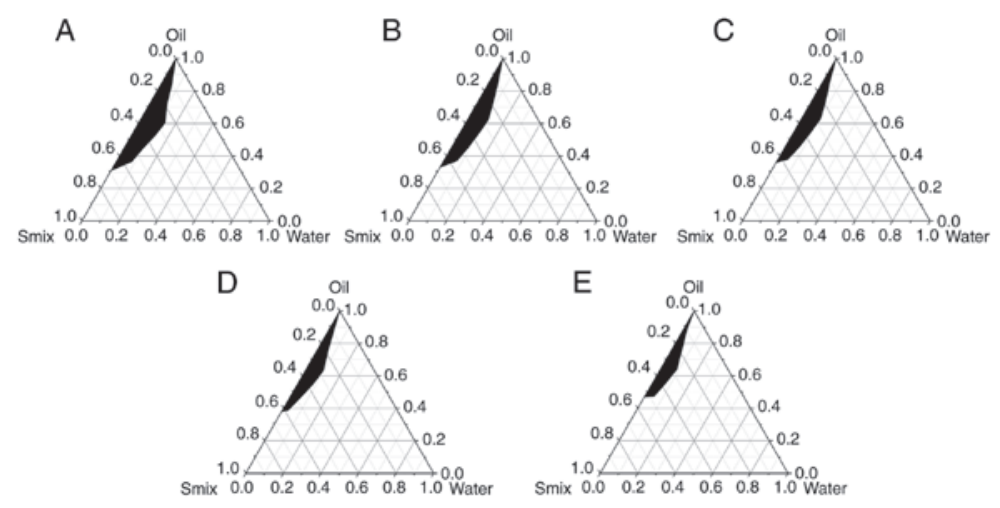

Figure 4. Pseudo-ternary phase diagrams using different concentrations of aqueous spirulina polysaccharide solution (A) $1 \%$; (B) $2 \%$; (C) $5 \%$; (D) $10 \%$; (E) $20 \%$. Smix, surfactant mix.

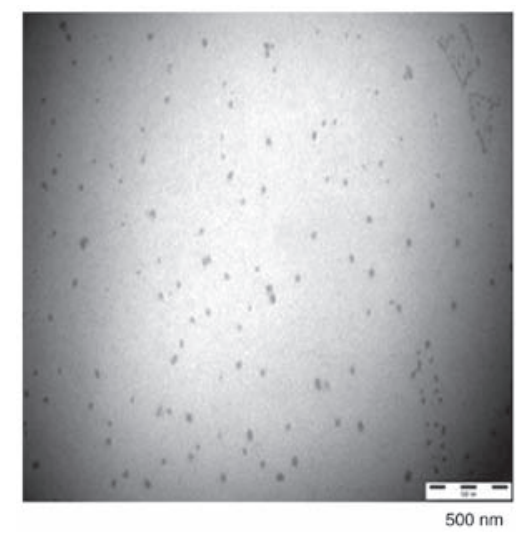

Figure 5. Transmission electron microscopy image of the spirulina polysaccharide nanoemulsion (magnification, x39,000). group, fluorescence was distributed throughout the body by $\sim 2 \mathrm{~h}$, mostly eliminated by $8 \mathrm{~h}$, and eliminated at $24 \mathrm{~h}$. By contrast, the fluorescence of the PSP-NE group was distributed throughout the body in $\sim 4 \mathrm{~h}$, and the fluorescence intensity was significantly higher than that of the PSP group. In addition, significant fluorescence accumulated in the liver and the kidney, and remained detectable at $24 \mathrm{~h}$, and almost eliminated at $36 \mathrm{~h}$. These findings indicated that the PSP-NE exhibited a sustained-release and tissue effect.

Antitumor assay. As shown in Fig. 8A and B, PSP-NE exhibited a superior inhibitory effect on MCF-7 cells $\left(\mathrm{IC}_{50}\right.$ at $500 \mu \mathrm{g} / \mathrm{ml}$ ) and HepG2 cells ( IC $_{50}$ at $\left.500 \mu \mathrm{g} / \mathrm{ml}\right)$, compared with PSP (MCF-7 cell IC $\mathrm{IC}_{50}$ at $1,000 \mu \mathrm{g} / \mathrm{ml}$; HepG2 cell $\mathrm{IC}_{50}$ at $2,000 \mu \mathrm{g} / \mathrm{ml})$. 


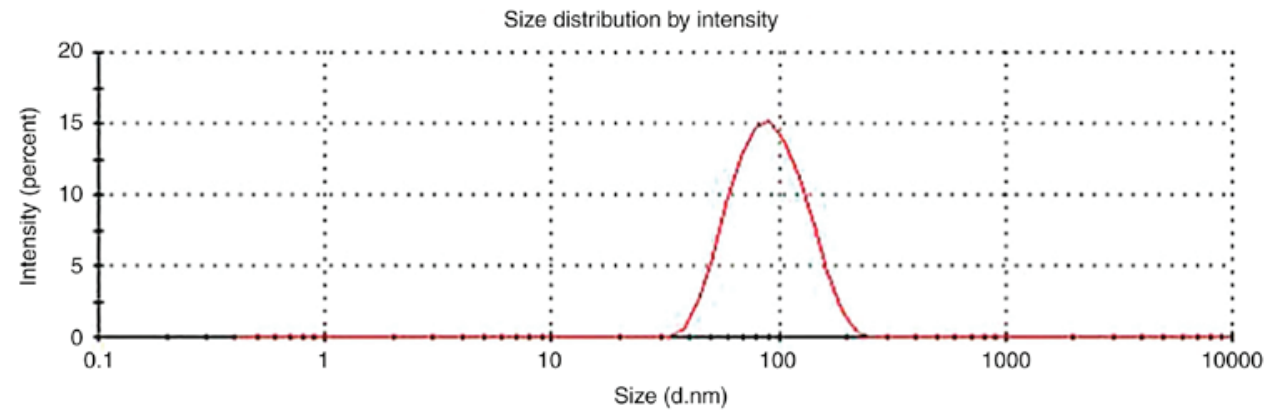

Figure 6. Particle size and PDI of the spirulina polysaccharide nanoemulsion.

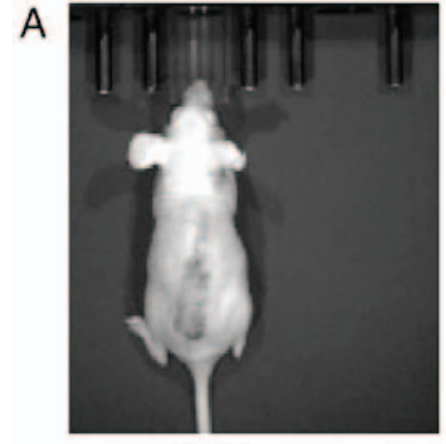

Empty

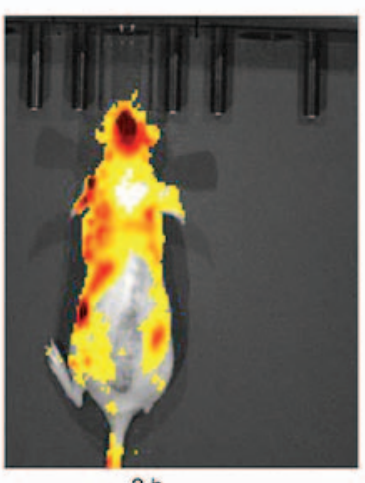

$2 \mathrm{~h}$

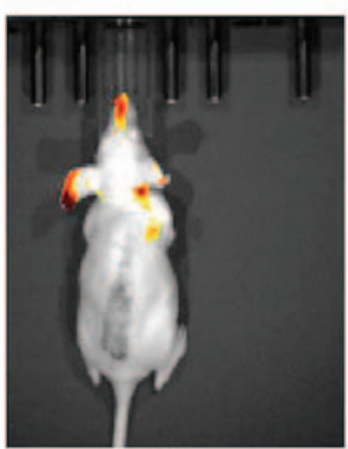

$24 \mathrm{~h}$

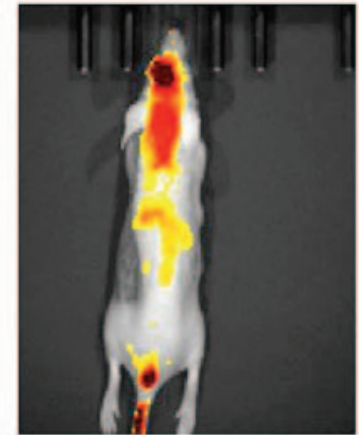

$0.5 \mathrm{~h}$

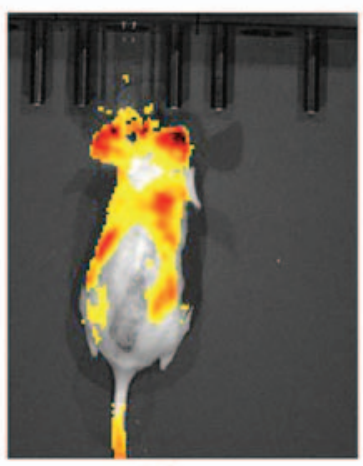

$4 \mathrm{~h}$

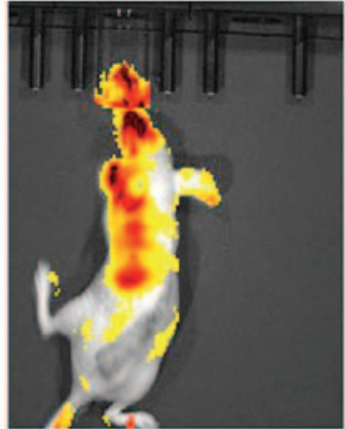

$1 \mathrm{~h}$

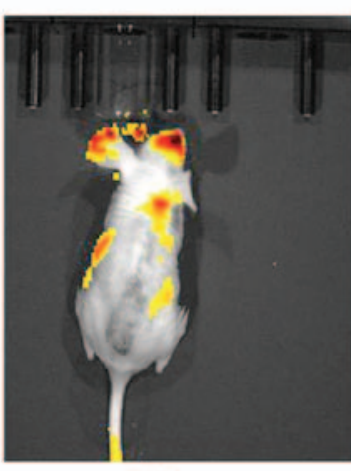

$8 \mathrm{~h}$

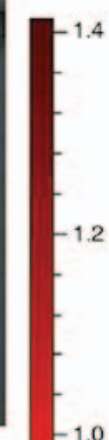

$-1.0$

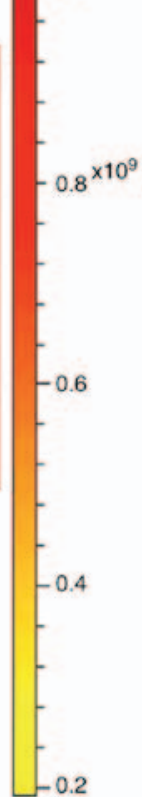

Radiant efficiency $\left(\frac{\mathrm{p} / \mathrm{sec} / \mathrm{cm}^{2} / \mathrm{s}}{\mu \mathrm{W} / \mathrm{cm}^{2}}\right)$

Color scale
Min $=1.91 \mathrm{e} 8$ $\operatorname{Max}=1.41 \mathrm{e} 9$

Figure 7. Distribution of PSP-NE. (A) Fluorescence in nude mice following intragastric administration of PSP and ICG at different time points. PSP, spirulina polysaccharide; NE, nanoemulsion; ICG, indocyanine green.

Antioxidant assay. In the present study, the potent free radical scavenging ability of PSP and PSP-NE on DPPH $\bullet$ and $\mathrm{OH} \bullet$ was determined at concentrations between 1 and $5 \mathrm{mg} / \mathrm{ml}$, which showed concentration-dependent effects. The scavenging abilities increased with increasing concentration. As shown in Fig. 9A and B, Vc exerted higher antioxidant scavenging of $\mathrm{DPPH} \bullet$, but a lower ability to scavenge $\mathrm{OH} \bullet$, compared with PSP or PSP-NE. However, no significant differences were 
B

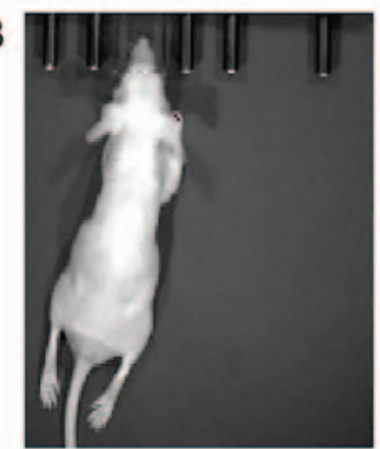

Empty

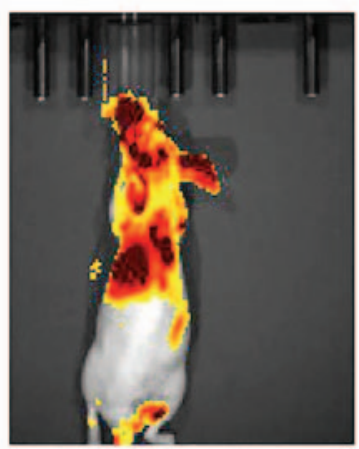

$2 \mathrm{~h}$

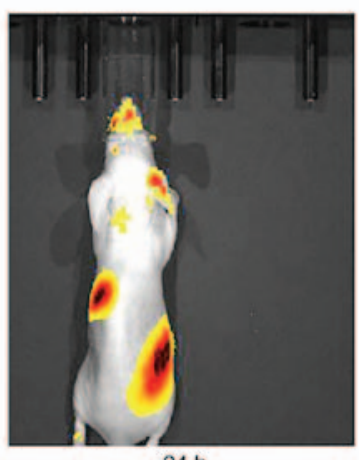

$24 \mathrm{~h}$

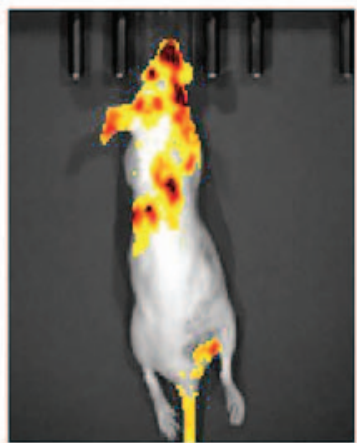

$0.5 \mathrm{~h}$

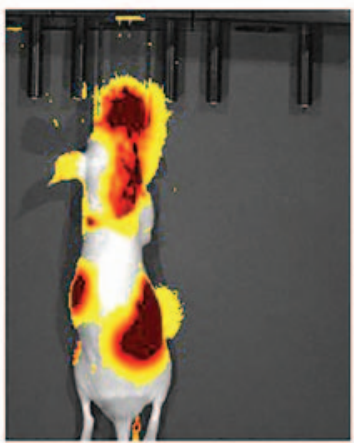

$4 \mathrm{~h}$

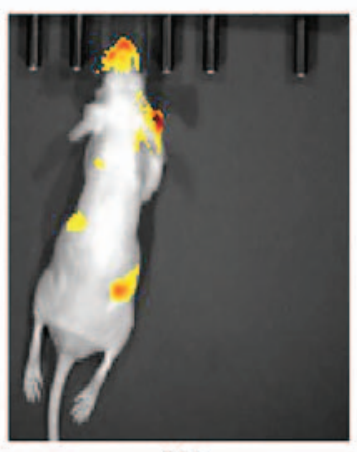

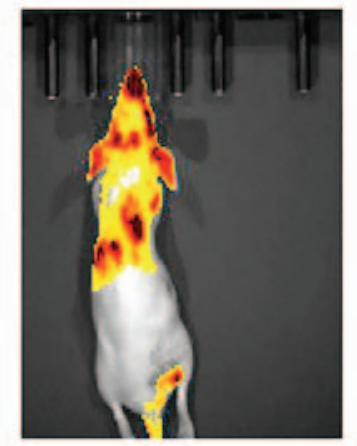

$1 \mathrm{~h}$

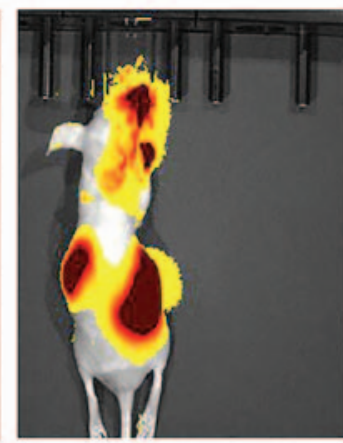

$8 \mathrm{~h}$

$36 \mathrm{~h}$

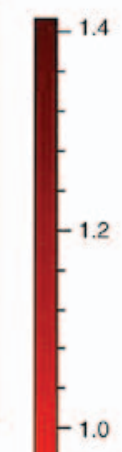

$-0.8^{\times 10^{9}}$

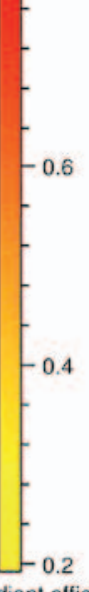

Radiant efficiency

$\left(\frac{\mathrm{p} / \mathrm{sec}^{\mathrm{cm}} \mathrm{cm}^{2} / \mathrm{s}}{\mu \mathrm{W} / \mathrm{cm}^{2}}\right)$

Color scale

Min=1.91e8

$\operatorname{Max}=1.41 \mathrm{e} 9$

Figure 7. Continued. Distribution of PSP-NE. (B) Fluorescence in nude mice following intragastric administration of PSP-ICG-NE at different time points. PSP, spirulina polysaccharide; NE, nanoemulsion; ICG, indocyanine green.

observed in the DPPH• and $\mathrm{OH} \bullet$ scavenging activity between PSP-NE and PSP at any concentrations.

\section{Discussion}

The surfactant screening process revealed that a single surfactant was not able to form an NE with superior properties. Therefore, using combinations of surfactants is suitable for the adjustment of HLB values and enhancement of emulsion stability. The HLB of Span 80 was 4.3 and that of Tween-80 was 15.0, and a 3:1 mixture ratio produced the desired HLB for soybean oil emulsification.

When the co-surfactant was screened, ethanol was selected, which presented with the largest NE formation area at a $K_{\mathrm{m}} 2: 1$. In the process of NE formation, liquid crystal and gel phenomenon (soybean phospholipids as a surfactant) may occur, and alcohol is conducive to the transition of a liquid crystal phase to an NE phase.

When screening the oil phase, the smaller the molecular volume, the higher the ability to dissolve the drug, and the faster the NE formation. Peanut and corn oils formed NE, but the resulting NE appeared turbid at high- or low-temperature storage (data not shown). Other oil phases not only formed NEs with good qualities but also remained unchanged at low and high temperatures. Therefore, liquid paraffin, sesame oil, injection-grade soybean oil, and medium-chain fatty acid triglycerides were selected as alternative oil phases. The screened mixed surfactants and co-surfactants were weighed according to the selected $K_{\mathrm{m}}$ value and mixed with the above oil phase, and the resulting formulation was screened, finally confirming that soybean oil was the optimal oil phase.

When the water phase was selected, the effects of PSP concentration on NE formation were observed, and the optimum PSP concentration was determined. The results showed that the PSP concentration within $20 \%$ did not markedly affect NE formation, and the NE became turbid at higher PSP concentrations. As polysaccharides are macromolecule materials, the area for NE formation gradually decreased at increased PSP concentrations. On considering the drug loading problem, $10 \%$ PSP aqueous solution was finally selected. 
Table II. Light properties of PSP-NE ( $n=3)$.

\begin{tabular}{rccccc}
\hline Time (days) & Appearance & Particle size & PSP content $(\mathrm{mg} / \mathrm{ml})$ & $\mathrm{pH}$ & $\begin{array}{c}\text { Viscosity } \\
\left.\text { (relative to water; }{ }^{\circ} \mathrm{E}\right)\end{array}$ \\
\hline 0 & Clear and transparent & $79.16 \pm 0.76$ & $5.17 \pm 0.03$ & $7.23 \pm 0.03$ & $11.52 \pm 0.04$ \\
5 & Clear and transparent & $79.42 \pm 0.65$ & $5.16 \pm 0.01$ & $7.21 \pm 0.00$ & $11.52 \pm 0.05$ \\
10 & Clear and transparent & $80.02 \pm 1.06$ & $5.16 \pm 0.03$ & $7.18 \pm 0.01$ & $11.55 \pm 0.05$ \\
\hline
\end{tabular}

PSP, spirulina polysaccharide; NE, nanoemulsion.

Table III. Accelerated test results of PSP-NE ( $n=3)$.

\begin{tabular}{|c|c|c|c|c|c|}
\hline $\begin{array}{l}\text { Time } \\
\text { (mins) }\end{array}$ & Appearance & Particle size & $\begin{array}{l}\text { PSP content } \\
(\mathrm{mg} / \mathrm{ml})\end{array}$ & $\mathrm{pH}$ & $\begin{array}{c}\text { Viscosity } \\
\left.\text { (relative to water; }{ }^{\circ} \mathrm{E}\right)\end{array}$ \\
\hline 0 & Clear and transparent & $78.63 \pm 0.50$ & $5.16 \pm 0.02$ & $7.24 \pm 0.01$ & $11.50 \pm 0.01$ \\
\hline 1 & Clear and transparent & $79.06 \pm 0.41$ & $5.15 \pm 0.01$ & $7.22 \pm 0.02$ & $11.49 \pm 0.03$ \\
\hline 2 & Clear and transparent & $79.66 \pm 0.46$ & $5.12 \pm 0.01$ & $7.20 \pm 0.02$ & $11.51 \pm 0.07$ \\
\hline 3 & Clear, transparent, slight darker color & $80.43 \pm 1.02$ & $5.10 \pm 0.02$ & $7.16 \pm 0.02$ & $11.53 \pm 0.02$ \\
\hline 6 & Clear, transparent, darker color & $80.75 \pm 1.30$ & $5.11 \pm 0.02$ & $7.14 \pm 0.03$ & $11.54 \pm 0.03$ \\
\hline
\end{tabular}

PSP, spirulina polysaccharide; NE, nanoemulsion.
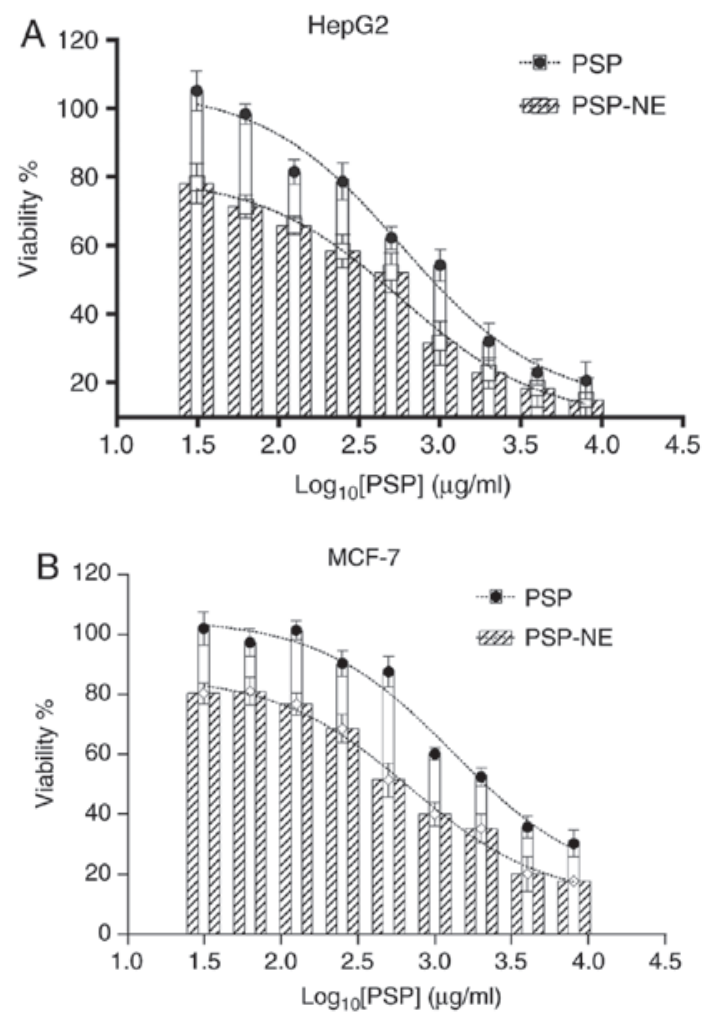

Figure 8. Cytotoxicity of PSP and PSP-NE. (A) In vitro cytotoxicity of PSP and PSP-NE against HepG2 cells. (B) In vitro cytotoxicity of PSP and PSP-NE against MCF-7 cells. PSP, spirulina polysaccharide; NE, nanoemulsion.

The majority of polysaccharides neither absorb light nor chromophores, making them difficult to quantify. However, the present study observed the distribution of the dosage form in
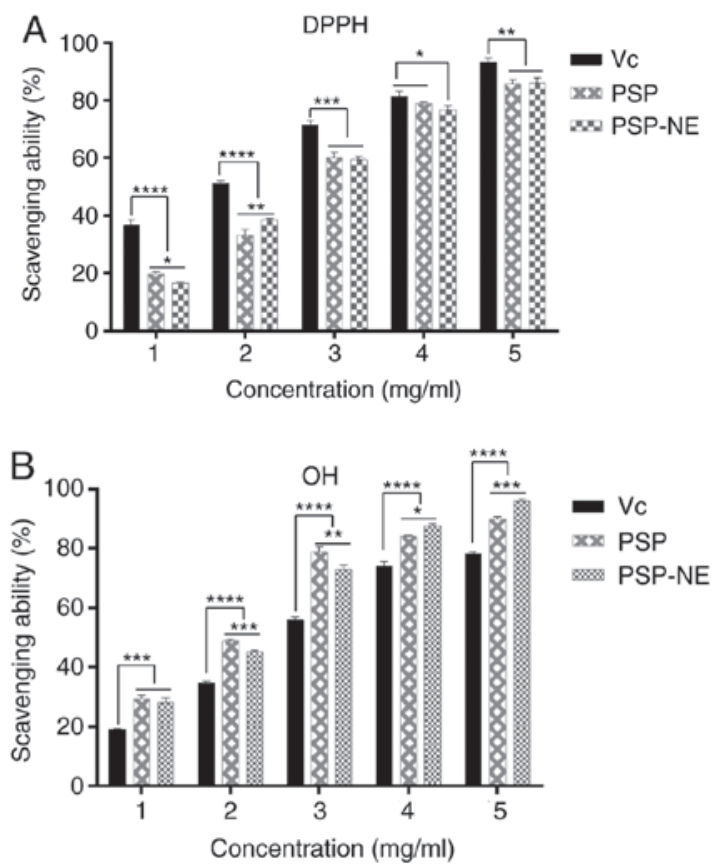

Figure 9. (A) Scavenging effect of PSP and PSP-NE on DPPH radicals compared with that of VC. (B) Scavenging activity of PSP and PSP-NE on $\mathrm{OH}$ radicals compared with that of VC. PSP, spirulina polysaccharide; NE, nanoemulsion; DPPH, 1 1,1-diphenyl-2-picrylhydrazyl; OH, hydroxyl radical; $\mathrm{VC}$, vitamin $\mathrm{C}$.

the body by using living imaging aids. In this experiment, ICG was embedded in PSP-NE, and the ICG-PSP and ICG-PSP-NE solutions were administered in nude mice in parallel. The body distribution of fluorescence was observed over time. The distribution and elimination rates of the PSP-treated group 
were faster than those of the PSP-NE group. These results indicated that the prepared NEs exhibited slow release.

The in vitro antitumor experiments on HepG2 and MCF-7 cells demonstrated that PSP-NE had a superior inhibitory effect compared with PSP, which may be attributed to the fact that PSP-NE increased the uptake of cells and increased the effective concentration of drugs.

It has been reported that PSP can affect the metabolism in the body (25). Ravi et al (26) demonstrated that PSP reduced the ratio of low-density lipoprotein to high-density lipoprotein (HDL), decreased the levels of plasma lipid, and modified the total cholesterol and HDL cholesterol levels. PSP has also been found to decrease the levels of postprandial blood glucose and glycosylated hemoglobin (HbAlc), and exhibit long-term glycemic regulation (27). Therefore, further investigation of the glucose and lipid metabolism of PSP-NE is required.

In conclusion, PSP-NE was prepared through a phase transition method, and the surfactant, co-surfactant, oil phase and water phase were screened through the use of a pseudo-ternary phase diagram. The prepared PSP-NE was transparent and uniform. Under TEM, the average particle size was $79.93 \pm 19 \mathrm{~nm}$; the PDI was $0.185 \pm 0.04$, PSP content was $5.14 \pm 0.06 \mathrm{mg} / \mathrm{ml}$, and the entrapment rate was $62 \%$. The PSP-NE did not exhibit demulsification in the stability assessment, indicating its stability. The live imaging distributions of PSP and PSP-NE in nude mice were observed with a small-animal live imaging instrument and fluorescein ICG. The distribution and elimination rates of the PSP-treated group were faster than those of the PSP-NE-treated group. Furthermore, the PSP-NE treated group exhibited marked liver and kidney accumulation, indicating that the release of NEs was sustained and specifically targeted to the tissue. The free radical scavenging activity and antitumor ability of PSP-NE were also analyzed. The antioxidant effect of PSP-NE was not affected but it exhibited enhanced antitumor ability. The in vitro experiments showed that PSP-NE had good antioxidant and antitumor abilities.

\section{Acknowledgements}

Not applicable.

\section{Funding}

This study was financially supported by the Ministry of Education in the New Century Excellent Talents (grant no. NECT-12-0677), the Science and Technology Program of Guangzhou (grant nos. 2014J4500005 and 201704030141), the Science Program of the Department of Education of Guangdong (grant no. 2015KGJHZ012) and the Special Project of International Scientific and Technological Cooperation in Guangzhou Development District (grant no. 2017GH16).

\section{Availability of data and materials}

All relevant materials and datasets are within the manuscript and are available.

\section{Authors' contributions}

BW performed the screening of PSP-NE formulation components, and was a major contributor in writing the manuscript; TC was responsible for the evaluation of $\mathrm{NE}$ quality; QL undertook the live imaging of distribution of PSP-NE in animals following oral administration; MD and QM performed the anti-tumor and anti-oxidant effects of PSP-NE; JCCW, RZ and LY made substantial contributions to acquisition, analysis and interpretation of data. JCCW, SPCC and YC were involved in drafting the manuscript and revising it critically for intellectual content. SPCC and YC made contributions to conception and design, and assisted in the analysis of all experiments. All authors read and approved the final manuscript.

\section{Ethics approval and consent to participate}

The protocol for the study was approved by the College of Pharmacy, Jinan University. The Laboratory Animal Ethics Committee of Jinan University approved all protocols (date of approval, 13/09/2016; certification no. 20160913003).

\section{Patient consent for publication}

Not applicable.

\section{Competing interests}

The authors declare that they have no competing interests.

\section{References}

1. Liu Q, Huang Y, Zhang R, Cai T and Cai Y: Medical application of spirulina platensis derived c-phycocyanin. Evid Based Complement Alternat Med 2016: 7803846, 2016.

2. Yang F, Tang Q, Zhong X, Bai Y, Chen T, Zhang Y, Li Y and Zheng W: Surface decoration by Spirulina polysaccharide enhances the cellular uptake and anticancer efficacy of selenium nanoparticles. Int J Nanomedicine 7: 835-844, 2012.

3. Kurd F and Samavati V: Water soluble polysaccharides from Spirulina platensis: Extraction and in vitro anti-cancer activity. Int J Biol Macromol 74: 498-506, 2015.

4. Capek P, Machová E and Turjan J: Scavenging and antioxidant activities of immunomodulating polysaccharides isolated from Salvia officinalis L. Int J Biol Macromol 44: 75-80, 2009.

5. Klaus A, Kozarski M, Niksic M, Jakovljevic D, Todorovic N and Van Griensven LJLD: Antioxidative activities and chemical characterization of polysaccharides extracted from the basidiomycete Schizophyllum commune. LWT-Food Sci Technol 44: 2005-2011, 2011.

6. Lai F, Wen Q, Li L, Wu H and Li X: Antioxidant activities of water-soluble polysaccharide extracted from mung bean (Vigna radiata L.) hull with ultrasonic assisted treatment. Carbohydr Polym 81: 323-329, 2010.

7. Chaiklahan R, Chirasuwan N, Triratana P, Loha V, Tia S and Bunnag B: Polysaccharide extraction from Spirulina sp. and its antioxidant capacity. Int J Biol Macromol 58: 73-78, 2013.

8. Balachandran P, Pugh ND, Ma G and Pasco DS: Toll-like receptor 2-dependent activation of monocytes by Spirulina polysaccharide and its immune enhancing action in mice. Int Immunopharmacol 6: 1808-1814, 2006.

9. Zhang H, Cui Y, Zhu S, Feng F and Zheng X: Characterization and antimicrobial activity of a pharmaceutical microemulsion. Int J Pharm 395: 154-160, 2010.

10. López-Quintela MA and Rivas J: Chemical reactions in microemulsions: A powerful method to obtain ultrafine particles. J Colloid Interface Sci 158: 446-451, 1993. 
11. Hoar TP and Schulman JH: Transparent water-in-oil dispersions: The oleopathic hydro-micelle. Nature 152: 201-203, 1943.

12. López-Quintela MA: Synthesis of nanomaterials in microemulsions: Formation mechanisms and growth control. Curr Opinion Colloid Interface Sci 8: 137-144, 2003.

13. Malik MA, Wani MY and Hashim MA: Microemulsion method: A novel route to synthesize organic and inorganic nanomaterials: 1st Nano Update. Arabian J Chem 5: 397-417, 2012.

14. Shafiq-un-Nabi S, Shakeel F, Talegaonkar S, Ali J, Baboota S, Ahuja A, Khar RK and Ali M: Formulation development and optimization using nanoemulsion technique: A technical note. AAPS PharmSciTech 8: Article 28, 2007.

15. Kong $M$ and Park HJ: Stability investigation of hyaluronic acid based nanoemulsion and its potential as transdermal carrier. Carbohydr Polym 83: 1303-1310, 2011.

16. Schmalfußa U, Neubert R and Wohlrab W: Modification of drug penetration into human skin using microemulsions. J Control Release 46: 279-285, 1997.

17. Sintov AC, Levy HV and Botner S: Systemic delivery of insulin via the nasal route using a new microemulsion system: In vitro and in vivo studies. J Control Release 148: 168-176, 2010.

18. Xavier-Junior FH, Huang N, Vachon JJ, Rehder VL, do Egito ES and Vauthier C: Match of solubility parameters between oil and surfactants as a rational approach for the formulation of microemulsion with a high dispersed volume of copaiba oil and low surfactant content. Pharm Res 33: 3031-3043, 2016.

19. Moghimipour E, Salimi A and Leis F: Preparation and evaluation of tretinoin microemulsion based on pseudo-ternary phase diagram. Adv Pharm Bull 2: 141-147, 2012.

20. Pons R, Carrera I, Caelles J, Rouch J and Panizza P: Formation and properties of mini-emulsions formed by microemulsions dilution. Adv Colloid Interface Sci 106: 129-146, 2003.
21. Roohinejad S, Oey I, Wen J, Lee SJ, Everett DW and Burritt DJ: Formulation of oil-in-water $\beta$-carotene microemulsions: Effect of oil type and fatty acid chain length. Food Chem 174: 270-278, 2015.

22. Li Q, Tiange C, Yinghong H, Ronghua Z, Susan C and Yu C: The preparation and evaluation of cepharanthine-nanostructured lipid carriers in vitro and in vivo. J Bio Tissue Eng 7: 848-857, 2017.

23. Yoo HS, Lee KH, Oh JE and Park TG: In vitro and in vivo anti-tumor activities of nanoparticles based on doxorubicin-PLGA conjugates. J Control Release 68: 419-431, 2000.

24. Wu X, Li R, Zhao Y and Liu Y: Separation of polysaccharides from Spirulina platensis by HSCCC with ethanol-ammonium sulfate ATPS and their antioxidant activities. Carbohydr Polym 173: 465-472, 2017.

25. González-Torres L, Vázquez-Velasco M, Olivero-David R Bastida S, Benedí J, González RR, González-Muñoz MJ and Sánchez-Muniz FJ: Glucomannan and glucomannan plus spirulina added to pork significantly block dietary cholesterol effects on lipoproteinemia, arylesterase activity, and CYP7A1 expression in Zucker fa/fa rats. J Physiol Biochem 71: 773-784, 2015.

26. Ravi M, De SL, Azharuddin S and Paul SFD: The benefcial effects of spirulina focusing on its immunomodulatory and antioxidant properties. Nut Diet Suppl 2: 73-83, 2010.

27. Parikh P, Mani U and Iyer U: Role of spirulina in the control of glycemia and lipidemia in type 2 diabetes mellitus. J Med Food 4: 193-199, 2001.

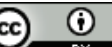

This work is licensed under a Creative Commons Attribution 4.0 International (CC BY 4.0) License. 